\title{
Mirror writing in a patient with Alzheimer disease
}

\section{HA de Silva ${ }^{1}$ and SB Gunatilake ${ }^{2}$}

\section{Introduction}

Mirror writing is "that variety of script which runs in an opposite direction to the normal, the individual letters also being reversed" [1]. Mirror writing may be transient or long lasting, and may affect single letters or characters, whole words or sentences. Although mirror writing occurs both spontaneously and pathologically, it is generally associated with cerebrovascular lesions in the dominant hemisphere [2].

\section{Case report}

We describe mirror writing in a 76-year old woman, a natural left-hander who had been forced by her parents to be right-handed. She had been able to mirror write with the left hand voluntarily during childhood. She presented with progressive impairment of recent memory of about 2 year's duration. She forgets names of friends, and gets disoriented in unfamiliar surroundings. She was well groomed, pleasant and oriented in time and place. Formal cognitive assessment with the Mini Mental State Examination (MMSE) and Cambridge Cognitive Examination (CAMCOG) showed impairment in short term recall and sustained attention associated with executive dysfunction. In addition, judgment was poor, and behaviour slightly disinhibited. Language ability, including wordfinding and fluency of expression, was normal. Episodic memory and semantic memory were normal. Visuospatial

Departments of ${ }^{1}$ Pharmacology and ${ }^{2}$ Medicine, Faculty of Medicine, University of Kelaniya, Sri Lanka.

Correspondence: HA de S, e-mail: <asita@mfac.kln.ac.lk> (Competing interests: none declared) Received 26 April 2005 and accepted 28 May 2005. 
normal and there was no evidence of constructional apraxia or agnosia.

She had no left-right disorientation and normally writes with the right hand. When she writes, she switches hands spontaneously and mirror writes with the left hand. This is seen frequently with both spontaneous and dictated writing (Figure 1). There were no localising features to suggest a focal brain lesion. There was no history of head trauma, cerebrovascular accident, or features of Parkinson disease. MRI scan of the brain showed only diffuse atrophy compatible with age. There was no focal atrophy affecting the hippocampal areas or the frontotemporal areas to suggest a specific dementia. Considering the clinical picture, the most probable diagnosis is early Alzheimer disease (AD).

\section{Discussion}

Mirror writing is seen in various disorders affecting the central nervous system. Acquired mirror-writing occurs most commonly in right-handed patients who use the left hand for writing following a right hemiplegia. Studies among the elderly in China and Japan have shown an increase in mirror writing in relation to cerebral damage and cognitive dysfunction [3, 4]. A high prevalence of mirror writing has been described in patients with essential tremor, Parkinson disease, and cerebellar disorders [2].

Mirror writing is also seen in people without any known disorder in the central nervous system. It is common as a normal stage when children first learn to write, but very few of them will continue to mirror write as adults [1], and persistent mirror writing is very rare in normal people. Leonardo da Vinci and Lewis Carroll are two well known people who wrote in both normal and mirror scripts [5]. Nevertheless, mirror writing in adults is probably more common than realised, and many with this ability are lefthanded. They would naturally adopt normal writing and may have their tendency to mirror write suppressed by others. The reason why mirror writing is usually carried out with the left hand has been attributed to abductive arm movements being easier and better coordinated than adductive movements. Leftward writing has therefore been held to be the natural direction of writing of the left-hander

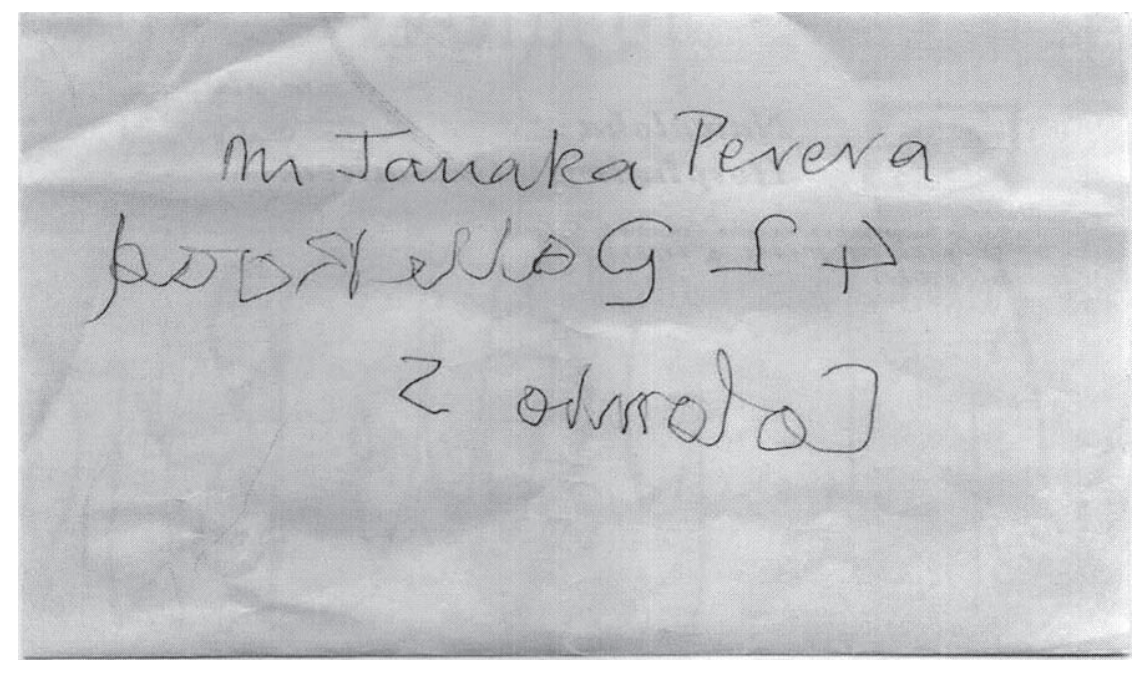

(a)

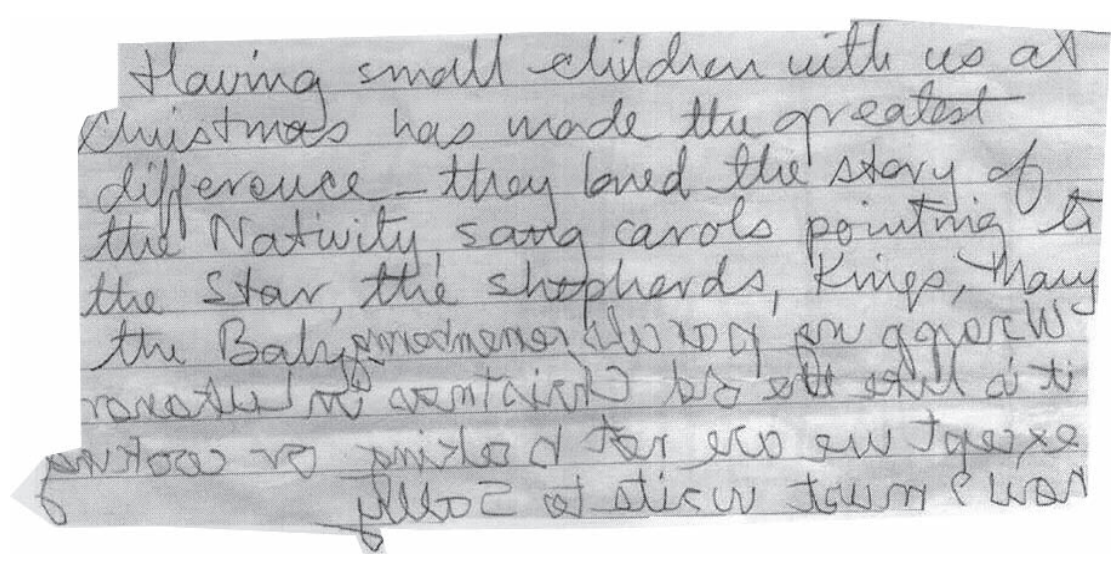

(b)

Figure 1. Mirror writing associated with dictated writing (a) and spontaneous writing (b) in this patient with probable AD. Note that during mirror writing the script moves leftward. 
$[1,5]$. More recently it has been observed that a surprisingly large number of reported left-handed mirror-writers are those whose native languages have traditionally been written and read leftward like Chinese, Japanese, and Hebrew languages [6]. When Chinese and English children were compared, the incidence of mirror-writing was significantly higher among Chinese children [7].

Acquired mirror-writing most commonly follows cerebrovascular lesions in various sites, usually in the left hemisphere. Pathological mirror-writing may occur without an identifiable lesion or after diffuse injuries [5]. The patient we describe fulfills diagnostic criteria [8] of probable AD. Her mirror-writing is probably a release phenomenon resulting in unmasking of a childhood habit caused by frontal disinhibition associated with AD.

\section{References}

1. Critchley M. Mirror-writing. London: Kegan Paul, Trench, Trubner, 1928.
2. Tashiro K, Matsumoto A, Hamada T, Moriwaka F. The aetiology of mirror writing: a new hypothesis. Journal of Neurology, Neurosurgery and Psychiatry 1987; 50: 1572-8.

3. Wang X, Cai X, Chen H. Mirror writing in the elderly. Chinese Medical Journal 1998; 111: 641-3.

4. Kuzuya M, Yamamoto T, Kuzuya F. Mirror writing in the aged. Nippon Ronen Igakkai Zashi 1991; 28: 499-503.

5. Schott GD. Mirror writing: Allen's self-observations, Lewis Carroll's "looking glass" letters, and Leonardo da Vinci's maps. Lancet 1999; 354: 2158-61.

6. Schott GD, Schott JM. Mirror writing, left-handedness, and leftward scripts. Archives of Neurology 2004; 61: 1849-51.

7. Wang XD. Mirror writing of Chinese characters in children and neurologic patients. Chinese Medical Journal 1992; 105: 306-11.

8. McKhann G, Drachman D, Folstein M, Katzman R, Price $\mathrm{D}$, et al. Clinical diagnosis of Alzheimer's disease: Report of the NINCDS-ADRDA work group. Neurology 1984; 34: 939-44. 